\title{
Research on the Crucial Pieces Consume Disciplinarian of Self-propelled Artillery Equipment
}

\author{
Ming Anqing, Lei Jie, Wang Jiancheng, Luo Hongfei, Liu Chunhua, Luo Liang \\ Wuhan Mechanical College, Wuhan Hubei, China
}

\begin{abstract}
The research on the crucial pieces consume disciplinarian of self-propelled artillery equipment have great significance for maintenance and support tasks. A crucial pieces impact factor is proposed and the consumption prediction model of crucial pieces is established based on the crucial pieces consumption factors analysis of self-propelled artillery in this paper. Application analysis shows that the model has high prediction accuracy and high universality which is fit for the crucial pieces consumption prediction of self-propelled artillery.
\end{abstract}

Keywords—crucial pieces, GM (1,1), impact factor

\section{自行火炮装备关重件消耗规律研究}

明安卿, 雷洁, 王建成, 罗鸿飞, 刘春华, 罗亮

武汉机械学院, 武汉, 湖北, 中国

\begin{abstract}
摘 要 研究自行火炮关重件消耗规律对做好维修保障任务具有重要意义。本文在分析自行火炮关重件消耗量影响因素的基础 上, 提出了关重件影响因子, 并建立了关重件消耗预测模型。应用分析表明, 该模型预测精度高, 通用性强, 适用于自行火炮关重件 消耗量预测。
\end{abstract}

关键词 关重件, $\mathrm{GM}(1,1)$, 影响因子

\section{1. 引言}

随着自动控制技术的不断发展, 新型自行火炮装备装 备现代化程度不断提高, 自行火炮关重件备件的品种和数 量也越来越多, 备件的保障费用在装备维修保障费用中占 的比例也越来越大。对新型自行火炮关重件消耗规律进行 分析, 科学合理地确定出自行火炮装备关重件储备数量, 并规划关重件供应工作成为亟待解决的难题[1]。也只有掌 握了自行火炮装备关重件消耗数量的有效规律, 才能正确 地加以运用, 以利于维修保障部门规划维修保障资源, 进 行费用估计, 并准确有效地完成维修保障任务。

长期以来, 自行火炮装备关重件备件需求量的预测, 主要以经验为主进行估计, 这种预测方法缺乏系统性和科 学性, 难以保证动态的备件需求, 不能对备件储备进行准 确的决策。为了使维修保障费效能发挥最大化, 本文在分 析影响关重件工作可靠性、寿命等因素对备件需求量影响 的基础上, 建立了自行火炮关重件备件需求预测模型, 为 新型武器装备备件的需求预测提供科学的依据。

\section{2. 关重件消耗量影响因素分析}

新型自行火炮装备关重件消耗量受到多种因素影响, 最直接的影响因素就是关重件自身的可靠性, 但又与使用 环境、训练强度等因素密切相关, 大体可分为四个方面。

由于生产工艺和关重件原材料的原因导致的关重件失 效, 属关重件固有的可靠性。部队全国分布, 装备的使用 和存放环境有很大差别, 高温潮湿和低温干燥的环境对关 重件的性能有很大影响, 环境越恶略, 关重件的性能越难 以发挥, 属使用环境因素。自行火炮装备使用时间和参加 重大军事演习次数的不同也决定着关重件的使用寿命, 而 装备在维护保养时关重件被多次拆卸安装会造成磨损、压 痕等也降低关重件的性能, 这两者属训练维护因素。最后 是人为因素, 不同驾驶员操作熟练程度不同, 由于人为的 误操作也会使关重件失效。

以上几个方面都影响着关重件的消耗量, 因此, 对关 重件备件数预测时, 必须要考虑它们的影响。 


\section{3. 关重件备件量预测模型}

\section{$3.1 \mathrm{GM}(1,1)$ 预测模型}

灰色理论是由华中理工大学邓聚龙教授于 1982 年首 次提出的。它是将原始数据进行变换处理, 通过处理后数 据随机性的弱化来研究发现表面上无序数据序列的潜在规 律[2]。最经典的灰色预测模型就是 $\operatorname{GM}(1,1)$ 模型, 其建模 和预测步骤如下:

根据收集到的关重件消耗数据, 构造原始时间序列:

$$
X^{(0)}=\left(x^{(0)}(1), x^{(0)}(2), \cdots, x^{(0)}(n)\right)
$$

式中, $x^{(0)}(k) \geq 0 \quad(k=1,2, \cdots, n)$ 。 对 $X^{(0)}$ 作一阶累加处理, 生成 $X^{(0)}$ 的 1-AGO 序列 ${ }^{[3]}$ :

$$
X^{(1)}=\left(x^{(1)}(1), x^{(1)}(2), \cdots, x^{(1)}(n)\right)
$$

式中, $x^{(1)}(k)=\sum_{i=1}^{k} x^{(0)}(i), k=1,2, \cdots, n$ 。

对 $X^{(1)}$ 作紧邻均值生成, 生成序列

$$
Z^{(1)}=\left(z^{(1)}(2), z^{(1)}(3), \cdots, z^{(1)}(n)\right)
$$

式中, $z^{(1)}(k)=\frac{\left(x^{(1)}(k)+x^{(1)}(k-1)\right)}{2} k=2,3, \cdots, n$ 。

作最小二乘估计, 得到 $\mathrm{GM}(1,1)$ 模型

$$
x^{(0)}(k)+a z^{(1)}(k)=b
$$

根据最小二乘估计参数满足:

$$
\hat{a}=(a, b)^{T}=\left(B^{T} B\right)^{-1} B^{T} Y
$$

式中

$$
Y=\left[\begin{array}{c}
x^{(0)}(2) \\
x^{(0)}(3) \\
\vdots \\
x^{(0)}(n)
\end{array}\right], B=\left[\begin{array}{c}
-z^{(1)}(2) \\
-z^{(1)}(3) \\
\vdots \\
-z^{(1)}(n)
\end{array}\right]
$$

解式(4)得 $\operatorname{GM}(1,1)$ 模型的时间响应式 ${ }^{[4]}$

$$
\hat{x}^{(1)}(t+1)=\left(x^{(1)}(0)-\frac{b}{a}\right) e^{-a t}+\frac{b}{a} \quad t=1,2, \cdots, n(5)
$$

通过式(7)得到估计值序列为

$$
\hat{X}^{(1)}=\left(\hat{x}^{(1)}(2), \hat{x}^{(1)}(3), \cdots, \hat{x}^{(1)}(n)\right)
$$

还原 $\hat{X}^{(1)}$ 得到原始序列的估计值序列为

$$
\hat{X}^{(0)}=\left(\hat{x}^{(0)}(2), \hat{x}^{(0)}(3), \cdots, \hat{x}^{(0)}(n)\right)
$$

以上即为灰色 $\operatorname{GM}(1,1)$ 模型的预测步骤, 从预测过程 可以看出若原始序列是在同一条件下得到的, 则 $\operatorname{GM}(1,1)$
模型可以得到比较满意的预测结果, 但从前文分析可知, 自行火炮关重件的性能和寿命与环境、人为因素相关性很 大, 而不同作战单位分布于不同的地域, 因此, 在进行建 模时, 需要在收集到的消耗数据的基础上加上前文分析因 素影响因子作为原始序列。

\section{2 关重件影响因子}

为了更直观的把环境、人为因素作为影响因子加入预 测, 需要对各个因素进行量化。根据分布地域的不同、操 作熟练度的不同等对影响因素进行分级, 从而可得各影响 因素量化后的数列 $t_{m}(n)$, 其中 $m$ 为影响因素个数, $n$ 该 影响因素的序号。

由于各影响因素单位和量级各不相同, 为了得到综合 影响因子, 采用下式分别对各影响因素进行无量纲化处理:

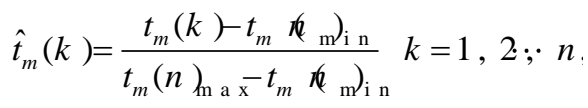

根据均方差公式定义关重件影响因子 $\delta$ 为

$$
\delta=\sqrt{\frac{1}{m-1} \sum_{j=1}^{m}\left(\hat{t}_{j}(n)-\overline{\hat{t}}_{j}(n)\right)^{2}}
$$

式中, $\overline{\hat{t}}_{j}(n)=\frac{1}{m} \sum_{j=1}^{m} \hat{t}_{j}(n)$ 。

上述影响因子主要针对某一年度因重大因素影响而使 数据产生突变时使用, 若在预测过程中没有重大因素, 而 且直接预测可满足精度要求, 可不必加入影响因子。

\section{3 预测模型的检验}

常用的灰色模型检验方法主要有四种: 平均相对误差 检验法、均方差比值检验法、关联度检验法、小误差概率 检验法 [5]。这四种检验方法都是通过残差来检验模型精度, 本文采用平均相对误差检验法来检验模型精度。

计算平均相对误差时, 须首先计算残差序列

$$
\partial^{(0)}=(\partial(1), \partial(2), \cdots, \partial(n))
$$

式中, $\partial(k)=x^{(0)}(k)-\hat{x}^{(0)}(k) \quad k=1,2, \cdots n$ 。

根据残差序列计算相对误差序列

$$
\nabla^{(0)}=(\nabla(1), \nabla(2), \cdots, \nabla(n))
$$

式中, $\nabla(k)=\left|\frac{\partial(k)}{x^{(0)}(k)}\right| k=1,2, \cdots n$ 。 
表 1 等级划分表

\begin{tabular}{|c|c|c|c|c|}
\hline 精度等级 & 一级 & 二级 & 三级 & 四级 \\
\hline 平均相对误差 & 0.01 & 0.05 & 0.10 & 0.20 \\
\hline
\end{tabular}

由以上两式可得平均相对误差 $\bar{\nabla}=\frac{1}{n} \sum_{k=1}^{n} \nabla(k)$ 。其等级 划分标准见表 1 。

用平均相对误差对预测模型进行检验时, 若算得模型 精度低于三级, 一般认为模型是不合理的, 需要对模型进 行修正, 而若检验精度在三级以上, 则认为模型合理。

\section{4. 应用分析}

通过部队调研, 得到某新型自行火炮的某种关重件 2007 年至 2011 年的消耗量统计数据如表 2 所示。本文首 先按照传统 GM $(1,1)$ 预测模型运用 Matlab 编程, 预测该关 重件的消耗量，并计算预测残差和相对误差。

表 2 关重件消耗量统计表

\begin{tabular}{|c|c|c|c|c|c|}
\hline 序号 & 1 & 2 & 3 & 4 & 5 \\
\hline 时间/年 & 2007 & 2008 & 2009 & 2010 & 2011 \\
\hline 消耗量/件 & 110 & 166 & 212 & 206 & 204 \\
\hline 预测值 & - & 181.84 & 191.58 & 201.86 & 212.68 \\
\hline 残差 & - & 15.84 & 20.42 & 4.14 & 8.68 \\
\hline 相对误差 & - & 0.095 & 0.096 & 0.020 & 0.043 \\
\hline
\end{tabular}

表 3 本文模型预测结果

\begin{tabular}{|c|c|c|c|c|c|}
\hline 序号 & 1 & 2 & 3 & 4 & 5 \\
\hline 时间/年 & 2007 & 2008 & 2009 & 2010 & 2011 \\
\hline 消耗量/件 & 110 & 206 & 212 & 206 & 204 \\
\hline 预测值 & - & 208.78 & 207.59 & 206.40 & 205.21 \\
\hline 残差 & - & 2.789 & 4.410 & 0.400 & 1.210 \\
\hline 相对误差 & - & 0.013 & 0.021 & 0.002 & 0.006 \\
\hline
\end{tabular}

由表 2 计算可得平均相对误差为 0.05 , 预测模型为二 级精度, 预测模型是合理的, 但通过调研发现, 2008 年的 总体训练时间相对较短, 而且训练时训练环境和气候也相 对较好, 因此, 在计算时, 应对 2008 年的消耗量加影响因 子。根据训练情况, 把时间分为 5 个等级, 把环境因素分 为 3 个等级, 根据式(10)、(11)计算可得影响因子为 $\delta=1.24$ 。将计算因子加入预测模型, 算得加入影响因子后 的消耗量、预测值、残差和相对误差如表 3 所示。

由表 3 计算可得平均相对误差为 0.01 , 预测模型为一 级精度。将两次预测值与原始数据进行比较, 可以看出预 测准确度较以前有很大提高, 模型预测性较好。

\section{5. 结论}

科学合理的自行火炮装备关重件备件数量是装备保障 费用能效发挥最大化的前提, 本文针对自行火炮装备关重 件消耗规律进行研究, 提出了关重件消耗影响因子, 建立 了关重件消耗数量预测模型, 将传统预测模型精度由二级 提高为一级, 大大提高了预测精度, 为确定自行火炮装备 关重件备件数量提供了依据。

\section{参考文献(References)}

[1] Zhang Lianghua, Shao Weidong, et al, "Study on equipment maintenance material consumption law based on multiplicative prediction model," Journal of Beijing Technology and Business University, vol. 28, no. 1, pp. 52-55, 2010.

[2] Deng Julong, "The gray theory," Wuhan: Huazhong University of Science Press, 2003.

[3] Ma Wenbo, and Guo Fuliang, "An application of grey-markov prediction model to fuel consumption," Ship Electronic Engineering, vol. 32, no. 6, pp. 106-107, 2012.

[4] Wang Fengxiao, and Zhou Weiping, "Statistical combination forecast method based on exponential smoothing and grey model," Journal of Kashgar Teachers College, vol. 33, no. 3, pp. $1-3,2012$.

[5] Liu Sifeng, Guo Tianbang, and Dang Yaoguo, "The grey system theory and its application,” Beijing: Science Press, 2000. 